\title{
Proposta de modelo para controle integrado da produção e da qualidade com apoio da computação móvel
}

\author{
An integrated production and quality control model \\ supported by mobile computing
}

\section{Cibeli Ferrando Leão \\ Eduardo Luis Isatto \\ Carlos Torres Formoso}

\section{Resumo \\ $\Delta$}

lguns estudos indicam que as perdas por making-do são frequentes na indústria da construção e constituem-se na causa-raiz de outras perdas, tais como acidentes, falhas de qualidade, retrabalho e trabalho em progresso. As perdas por making-do referem-se à situação na qual uma tarefa começa ou continua sem ter disponíveis todos os itens necessários para sua conclusão. Há evidências de que as perdas por making-do estão também relacionadas com a falta de terminalidade das tarefas e com a execução de pacotes de trabalho informais. A literatura sugere que a integração entre os controles da produção e da qualidade é um meio de reduzir a incidência de pacotes informais e, consequentemente, as perdas por making-do. Para viabilizar o desenvolvimento de sistemas de controle que permitam coletar e processar dados sobre esses problemas de forma eficiente, é necessário explorar o uso da computação móvel, que oferece oportunidades de inovação em sistemas de controle. Assim, o objetivo principal deste estudo é desenvolver um modelo de controle integrado da produção e da qualidade, vinculado ao Sistema Last Planner, com o apoio da computação móvel. Foi realizado um estudo empírico em dois empreendimentos habitacionais de baixa renda, no qual o modelo proposto foi avaliado. A aplicação do modelo permitiu coletar dados relacionados à conclusão de pacotes de trabalho, execução de pacotes informais, falta de terminalidade das tarefas, perdas por making-do e verificação da qualidade.

Palavras-chaves: Controle integrado. Gestão da produção. Qualidade. Perdas. Computação móvel.

Cibeli Ferrando Leão Centro Universitário Ritter dos Reis Porto Alegre - RS - Brasil

Eduardo Luis Isatto Universidade Federal do Rio Grande do Sul Porto Alegre - RS - Brasil

Carlos Torres Formoso Universidade Federal do Rio Grande do Sul Porto Alegre - RS - Brasil

Recebido em 28/02/15 Aceito em 16/10/15

\section{Abstract}

A number of studies have pointed out that making-do is a frequent type of waste in the construction industry and is the root cause of other wastes, such as accidents, quality failures, rework and work in progress. Making-do waste refers to a situation in which a task starts or continues without having available all the inputs required for its completion. There are evidences that making-do waste is strongly related to unfinished work and the execution of informal work packages. The literature suggests that the integration between production and quality control is a means for reducing the incidence of informal packages and, consequently, makingdo. The use of mobile devices in construction sites is considered an efficient alternative to collect and manage information, providing opportunities to innovate in production control. The aim of this study is to devise a production and quality control model, linked to the Last Planner System, using the support of mobile computing. An empirical study was conducted in two low-cost housing projects, in which the proposed model was evaluated. The implementation of the model allowed the collection of data related to work package completions, execution of informal packages, unfinished work, making-do wastes and quality checks.

Keywords: Integrated control. Production management. Quality. Waste. Mobile computing. 


\section{Introdução}

Diversos estudos, realizados em diferentes países, indicam que as perdas na construção civil representam uma percentagem relativamente alta dos custos da produção (FORMOSO et al., 2002; HWANG et al.; 2009; KOUSHKI; KARTAM, 2004; LOVE; LI, 2000). Buscando atuar nas causas das perdas na construção civil, Koskela (2004) propõe a consideração de uma nova categoria de perda, denominada making-do ${ }^{1}$. A perda por making-do pode ser definida como a redução de desempenho que ocorre quando uma tarefa é iniciada sem que todos os recursos necessários para sua realização estejam disponíveis ou quando a tarefa continua a ser executada diante da indisponibilidade de algum recurso. Tais perdas têm sido apontadas como a causa-raiz de outras perdas na produção, tais como acidentes, problemas de qualidade, retrabalho e excesso de trabalho em progresso (FIREMAN; FORMOSO; ISATTO, 2013; FORMOSO et al., 2011).

Embora o número de estudos que mediram perdas por making-do ainda seja pequeno (BONESI, 2014; FIREMAN, 2012; SILVA, 2013; SOMMER, 2010; VELOSO, 2014), há indicações de que essas perdas são bastante frequentes. Evidências indiretas também sugerem uma grande incidência de making-do na construção civil: diversos estudos sobre a implementação do

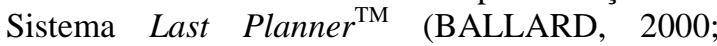
FORMOSO; MOURA, 2009; MACHADO, 2003; SANTOS, 2004) apontaram que uma das causas mais importantes das falhas de planejamento é a gestão ineficaz de fluxos a montante (por exemplo, fornecimento de materiais, projeto, equipamentos, entre outros).

Ao investigar as perdas por making-do que ocorrem nos canteiros de obra, Fireman, Formoso e Isatto (2013) identificaram sua associação com a falta de terminalidade das tarefas. Por terminalidade entende-se a conclusão das tarefas no prazo e com a qualidade determinada, sem a necessidade de retorno posterior de alguma equipe para a realização de retrabalhos ou arremates (ALVES, 2000). Quando há falta de terminalidade nas tarefas, sua efetiva conclusão, na maioria das vezes, não é planejada, resultando em tarefas informais, sujeitas a incertezas e perdas por making-do (FIREMAN; FORMOSO; ISATTO, 2013).

\footnotetext{
${ }^{1}$ Making-do é um conceito utilizado para definir uma situação na qual as tarefas são realizadas com aquilo que se tem disponível, ou, em uma linguagem mais coloquial, significa "dar um jeitinho" (SOMMER, 2010). Assim, por não existir uma tradução exata para o português, optou-se em manter o termo em inglês.
}

Fireman (2012) propôs um método de controle integrado da produção e da qualidade, com ênfase na medição de perdas por making-do e retrabalho. A implementação desse método de controle indicou ser possível reduzir as perdas por making $d o$ a partir da integração entre os sistemas de controle da produção e da qualidade. No entanto, ao avaliar o método de controle quanto a sua aplicabilidade, Fireman (2012) observou que era despendido um longo tempo para as atividades de coleta, entrada de dados e processamento dos dados em si. Dessa forma, foi sugerido pelo mesmo autor o emprego da computação móvel para acelerar a coleta e o processamento de dados.

A literatura apresenta diversas pesquisas que implementaram o uso de computação móvel na construção civil, apontando a utilidade desse tipo de tecnologia (KIMOTO et al., 2005; KIM et al., 2008, 2013; NOURBAKHSH et al., 2012). Tais estudos indicam que o uso de dispositivos móveis no canteiro de obras tem forte potencial para melhorar o nível de compartilhamento de dados e as práticas de comunicação na indústria da construção, representando oportunidades para inovar os processos existentes de gestão da construção.

Assim, o objetivo principal deste estudo é desenvolver um modelo de controle integrado da produção e da qualidade, com o apoio de computação móvel. Optou-se vincular esse modelo de controle ao Last Planner ${ }^{\mathrm{TM}}$ pelo impacto positivo que este sistema tem na gestão da produção e também pelo fato de que este sistema possui mecanismos para reduzir a ocorrência de making-do por meio da identificação e remoção sistemática das restrições (BALLARD, 2000; FORMOSO; MOURA, 2009).

\section{Controle integrado da produção e da qualidade}

De acordo com Laufer e Tucker (1987), controlar envolve medir e avaliar o desempenho, assim como realizar ações corretivas quando o desempenho diverge do planejado. Segundo Ballard e Howell (1998), para que o controle tenha um papel proativo, auxiliando na identificação e na correção das causas dos problemas, deve ser realizado em tempo real, agindo diretamente na realização dos processos de produção, expandindose para além do papel de inspeção ou verificação. A falta de um controle proativo no nível da unidade de produção aumenta a incerteza na construção e impede que o planejamento seja

110 Leão, C. F.; Isatto, E. L.; Formoso, C. T. 
utilizado como uma ferramenta para moldar o futuro (BALLARD, 2000).

Segundo Koskela (1999), para que possa haver melhorias no sistema de produção, é preciso identificar a fonte da variabilidade, realizar as ações corretivas necessárias e monitorar o impacto dela. O Sistema Last Planner $^{\mathrm{TM}}$ (LPS), proposto por Ballard (2000), busca atender a esses princípios básicos combinando as funções controle e melhoria, de forma a reduzir a variabilidade e as perdas resultantes dela (KOSKELA, 1999). Koskela (2004) afirma que a causa principal de making-do é a variabilidade da produção, sendo aplicado quando há uma indisponibilidade inesperada de algum recurso. Essa indisponibilidade de recursos pode resultar em dois tipos de efeitos: causar a interrupção do trabalho ou gerar o surgimento de improvisações (FORMOSO et al., 2011).

Uma das dificuldades de aplicação prática do conceito de making-do reside no fato de que sua ocorrência está associada à falta de um recurso, não sendo, portanto, fácil de se observar diretamente. No entanto, indícios de sua ocorrência tornam-se visíveis através das situações de improvisação originadas a partir de situações de making-do. Segundo Formoso et al. (2011), making-do e improvisação são dois conceitos fortemente relacionados, uma vez que, ao se deparar com alguma dificuldade ou incerteza, as pessoas costumam utilizar qualquer recurso que esteja a seu alcance para conseguir atingir seus objetivos, ou os objetivos podem ser redefinidos a partir dos recursos disponíveis.

Porém, nem toda situação de improvisação está associada com making-do. Visando estabelecer um mecanismo para identificação de perdas por making-do, Sommer (2010) avança em relação ao tema propondo um método de identificação e mensuração das perdas por improvisação associadas com making-do na construção civil. Tomando por base observações realizadas no canteiro de obras, a autora agrupou as ocorrências dessas improvisações em sete categorias, com base no tipo de solução empregada: "acesso e mobilidade", "ajuste de componentes", “área de trabalho", "armazenamento", "equipamentos e ferramentas", "instalações provisórias" e "proteção". Fireman (2012) ainda propôs a inclusão de uma oitava categoria de improvisação, denominada de "sequenciamento", referindo-se à alteração na ordem de produção ou rearranjo da sequência de ataque a qual equipes de produção recorrem buscando evitar a interrupção de seu trabalho em virtude da indisponibilidade de um recurso ou tarefa precedente.
Machado (2003) e Santos (2004) também apontaram a importância de analisar sistematicamente as restrições, antecipando e planejando os recursos necessários para a execução das tarefas. As antecipações gerenciais propostas por Machado (2003) são constituídas por ações gerenciais referentes ao provimento dos recursos necessários para a execução dos serviços. Segundo este último autor, essas antecipações podem ser originadas do conhecimento adquirido em projetos anteriores e deveriam ser incluídas no processo hierarquizado de planejamento da produção.

Já Santos (2004) propôs o conceito de atividades facilitadoras, definidas como atividades que, ao serem colocadas no sistema de produção, são capazes de impedir ou minimizar possíveis interrupções ao longo do processo de produção, e podem apresentar-se através de antecipações ou de remoção de restrições. As atividades facilitadoras podem apresentar-se como atividades incorporadas no processo, atividades de apoio à produção ou, ainda, como informações, sendo classificadas em nove categorias: acesso, projeto, preparação do trabalho, conferência do trabalho, conflito espacial, sequenciamento, proteção dos operários, proteção dos processos e programação de obra (SANTOS, 2004).

É possível identificar semelhanças entre as categorias de atividades propostas por Machado (2003) e Santos (2004) e as categorias de perdas por improvisações apresentadas por Sommer (2010). A diferença está na finalidade para a qual elas foram propostas (SOMMER, 2010). Enquanto Machado (2003) e Santos (2004) propõem uma lista de ações que buscam evitar interrupções na produção, Sommer (2010) apresenta as alternativas encontradas pelos operários para não deixar que essas interrupções ocorram.

Em relação ao controle da qualidade, Picchi e Agopyan (1993) apontam que os serviços da obra devem ser controlados de forma sistemática, baseados em listas de verificação que estabeleçam os itens a ser avaliados e suas respectivas tolerâncias. Para isso, antes da realização de cada serviço, deve ser feita uma verificação dos recursos necessários para o bom andamento do serviço e também deve ser redigido um procedimento de execução que descreva detalhadamente como ele deve ser realizado (PICCHI; AGOPYAN, 1993). Segundo Misfeldt e Bonke (2004), a qualidade deve ser controlada e aprovada antes de uma atividade ser dada como concluída, a fim de garantir que as tarefas subsequentes não serão executadas sobre partes da construção que apresentam defeitos. 
Segundo Marosszeky et al. (2002), muitas empresas possuem sistemas de controle de qualidade abrangentes e bem documentados, porém, em muitos casos, são concebidos para satisfazer os requisitos administrativos do sistema de gestão em vez de melhorar a qualidade do produto para o cliente. Soares (2003) sugere que o sistema de PCP poderia ser utilizado como um meio para a implementação de algumas características importantes para o sistema de gestão da qualidade, como o envolvimento dos funcionários, a busca pela melhoria contínua, a utilização de indicadores, a padronização de processos e o foco na satisfação dos clientes internos e externos. Em vez de métricas de desempenho, o controle de qualidade deveria ser focado na identificação dos desvios que ocorrem durante a execução das tarefas e no conhecimento de suas causas (KOSKELA; HOWELL, 2002).

Buscando a melhoria desses sistemas de gestão e a redução das não conformidades, Sukster (2005) propôs diretrizes para a integração dos sistemas de controle da produção e da qualidade:

(a) a realização de reuniões periódicas de integração dos dois sistemas;

(b) a utilização conjunta de procedimentos do sistema de gestão da qualidade nos planos de médio e curto prazo;

(c) a inclusão do planejamento e controle da produção dentro do sistema de gestão da qualidade;

(d) a utilização de indicadores para avaliar aspectos de ambos os sistemas; e

(e) a criação de mecanismos para aumentar a participação da equipe no planejamento e controle dos serviços.

Ao longo do estudo em que propõe um método de controle integrado da produção e da qualidade, Fireman (2012) salienta a necessidade de identificação da causa-raiz, pois muitas vezes a falta de qualidade é decorrente da propagação de problemas da tarefa precedente (FIREMAN, 2012). Nesse sentido, ele chama a atenção para o fato de que muitos dos problemas identificados são causados pela indisponibilidade de pré-requisitos, sugerindo que o método também possa contribuir para o controle das perdas pela possibilidade de identificação de ocorrências do fenômeno de making-do durante a produção (FIREMAN, 2012).

Sommer (2010) também analisou as causas que deram origem às perdas por making-do durante seu estudo, chamando-as de "natureza das perdas por making-do". O critério proposto para classificar as perdas segundo sua origem foi baseado no tipo de recursos ou condição cuja indisponibilidade causa a situação de making-do: "informação", "materiais e componentes", "mão de obra", "equipamentos ou ferramentas", "espaço", "serviços interdependentes (atividades predecessoras)", "condições externas" e "instalações" (SOMMER, 2010).

\section{Sistema Last Planner}

O LPS apresenta duas funções principais: o controle da unidade de produção e o controle de fluxo do trabalho. A primeira função visa fazer melhores atribuições para os trabalhadores através da aprendizagem contínua e de ações corretivas. Já a segunda função está voltada em fazer com que o fluxo de trabalho siga através da unidade de produção na melhor sequência possível (BALLARD, 2000). O LPS pode ser considerado como um sistema de produção puxada, segundo definição proposta por Hopp e Spearman (1996): o sistema puxado autoriza a realização de uma tarefa a partir do status do sistema, em vez de estimativas de demanda.

O LPS, em geral, é dividido em três níveis hierárquicos. O planejamento de longo prazo, também chamado de plano-mestre, é um cronograma desenvolvido antes de se iniciar a construção, que descreve o trabalho a ser realizado durante toda a duração da obra (BALLARD, 2000). Esse plano deve ter um baixo grau de detalhamento, devido às incertezas relacionadas às durações reais e às entregas (BALLARD, 2000). O planejamento de médio prazo, chamado por Ballard (2000) de lookahead planning, é um planejamento móvel que tem como horizonte algumas semanas à frente, dando atenção às tarefas que supostamente acontecerão em determinado período e identificando quais ações serão necessárias para permitir a realização dessas tarefas no período desejado (BALLARD, 2000). Eventuais restrições à execução dos pacotes de trabalho identificadas acarretam a indisponibilidade do pacote de trabalho para as equipes de execução até que tais restrições sejam removidas, evitando, assim, a programação de pacotes de trabalho que não contem com os requisitos básicos para sua execução integral. Assim, no plano de médio prazo é criado um estoque de atividades aptas a ser realizadas, a partir do qual é formado o plano de trabalho semanal, ou plano de curto prazo.

No plano de curto prazo são definidos os pacotes que serão efetivamente executados a cada semana (BALLARD, 2000), a partir dos pacotes de trabalho disponibilizados pelo planejamento de médio prazo (portanto, sem restrições a sua execução). 
No LPS o pacote de trabalho (assignment) é considerado como um trabalho específico que determinada equipe compromete-se a realizar (BALLARD, 2000). Assim, o pacote de trabalho deve ser suficientemente detalhado, a fim de permitir que seja preparado e que sua conclusão possa ser identificada claramente (BALLARD; HOWELL, 1998; BALLARD, 2000). Para tanto, Marchesan (2001) sugere que um pacote de trabalho seja definido como o resultado de uma ação aplicada sobre determinado elemento, em determinado local.

Esse pacote de trabalho deve ser concebido formalmente no planejamento de curto prazo. No entanto, no controle da produção podem ser identificadas situações nas quais os trabalhadores ou equipes estão realizando atividades não planejadas para o período, sendo estas denominadas de pacotes informais. Segundo Fireman (2012), os pacotes informais podem ser de três naturezas:

(a) novos, ou seja, sua execução não foi autorizada pelo sistema de PCP;

(b) por falta de terminalidade, ou seja, refere-se à conclusão de um pacote que não foi integralmente executado no ciclo anterior; e

(c) de retrabalho, ou seja, causados pela falta de qualidade dos pacotes considerados como concluídos.

Com o LPS é possível avaliar a confiabilidade dos planos de trabalho, a partir do indicador denominado PPC (porcentagem de pacotes concluídos), que é calculado dividindo-se o número de pacotes de trabalho concluídos pelo número total de pacotes planejados (BALLARD, 2000). No entanto, o indicador PPC considera apenas a conclusão dos pacotes de trabalho, mas não sua qualidade (SUKSTER, 2005; MAROSSZEKY et al., 2002) . Assim, foram propostos por Sukster (2005) novos indicadores para avaliar a qualidade dos pacotes de trabalho conforme os critérios especificados no sistema de gestão da qualidade: PPCQ (porcentagem de pacotes concluídos com qualidade) e PPCR (porcentagem real dos pacotes concluídos).

Eventualmente, um pacote de trabalho pode ser considerado concluído no cálculo do PPC, mas são deixadas para trás pequenas tarefas de acabamento, sendo necessário o retorno de alguma equipe para concluir o trabalho ou para realizar algum tipo de correção ou retrabalho (SUKSTER, 2005; FIREMAN, 2012). Considerando que a terminalidade se refere à conclusão das tarefas no prazo com a qualidade determinada, sem a necessidade de retorno posterior para a realização de retrabalhos ou arremates (ALVES, 2000), a presente pesquisa considera apenas duas categorias de pacotes informais, sendo os pacotes de retrabalho integrados à categoria falta de terminalidade, conforme sugerido por Fireman, Formoso e Isatto (2013).

$\mathrm{Yu}$ et al. (2009) sugerem que os pacotes de trabalho devem ser verificados em sua origem, e todo reparo necessário deve ser realizado imediatamente, antes de os trabalhadores deixarem o local (YU et al., 2009). Fireman (2012) sugere que, quando um pacote de trabalho for reprovado pelo sistema de qualidade, ele deva ser corrigido de uma das seguintes maneiras: se ainda houver tempo na semana, o retrabalho seja realizado como um pacote informal; ou, se não houver tempo, o pacote de retrabalho seja incluído formalmente no planejamento semanal da semana seguinte.

\section{Método de pesquisa}

A abordagem metodológica adotada para este trabalho foi o design science research (DSR) ${ }^{2}$, que é uma forma de produção de conhecimento, segundo Lukka (2003), usada para produzir artefatos inovadores com o objetivo de solucionar problemas do mundo real e dar uma contribuição para a teoria da disciplina na qual está sendo aplicada. Tem-se como objetivo criar um artefato para solucionar um problema do mundo real, ou seja, propor um modelo de controle integrado da produção e da qualidade, além de implementar esse artefato na prática, para avaliar sua utilidade.

O modelo de controle integrado foi desenvolvido e testado no canteiro de obra de dois empreendimentos horizontais similares de uma empresa construtora de Porto Alegre, RS, financiados pelo Programa Minha Casa Minha Vida (PMCMV).

\section{Sistemas de gestão da empresa}

A empresa possui um procedimento gerencial que estabelece diretrizes para o planejamento e o controle da produção das atividades realizadas na obra. Conforme descrito no procedimento, o processo de PCP inicia-se com o cronogramamestre, desenvolvido pelo setor de planejamento da empresa, e depois deve seguir as etapas de planejamento de médio prazo, identificação e remoção de restrições, definição do plano semanal e, ao término de cada semana, a verificação dos serviços executados e o cálculo do PPC.

${ }^{2}$ Pode ser traduzido por "pesquisa nas ciências do design". Entretanto, adotou-se neste artigo a designação em inglês, uma vez que não existe ainda uma tradução amplamente usada em português. 
A partir das observações realizadas no canteiro de obras durante o estudo, constatou-se a utilização e atualização do cronograma-mestre e a realização semanal das reuniões de curto prazo. Porém, não existe uma rotina de reuniões de médio prazo, nas quais deveria ocorrer a identificação, análise e remoção de restrições.

Quanto ao sistema de gestão da qualidade, esse é descrito no Manual da Qualidade, sendo baseado nas normas ISO 9001/2008 e no SiAC do PBQP-H nível A. Para a etapa da produção são utilizados dois tipos de planilhas de verificação da qualidade: as PVQ (planilhas de verificação da qualidade), utilizadas para verificar a qualidade na execução dos serviços, e a Planilha de Avaliação Mensal, que avalia o sistema gerencial da obra como um todo. Nas PVQs itens específicos dos serviços que estão sendo realizados devem ser avaliados, sendo considerados aprovados ou reprovados. Devido ao fato de o preenchimento da PVQ ser demorado e exigir concentração, essa atividade costuma ser postergada para a liberação de outro serviço, funcionando como uma verificação de pré-serviço. Assim, os pacotes de trabalho concluídos normalmente não são avaliados quanto a sua qualidade ao final da semana.

\section{Etapas do estudo empírico}

O estudo empírico foi dividido em quatro etapas. $\mathrm{Na}$ primeira etapa foi desenvolvida uma ferramenta para coleta de dados baseada no método de controle integrado proposto por Fireman (2012). Foram utilizadas planilhas digitais, acessadas através de tablets, possibilitando a coleta de dados relacionados à ocorrência de perdas por making-do, execução de pacotes informais e conclusão de pacotes planejados.

A segunda etapa do estudo empírico foi focada no desenvolvimento de uma nova versão do modelo de controle integrado da produção e da qualidade, sendo propostas melhorias para a versão inicial elaborada na primeira etapa. Para isso foram consideradas as dificuldades encontradas na aplicação da primeira versão do modelo e as possibilidades de melhorias identificadas. O principal desafio era integrar efetivamente o controle da produção e da qualidade, com o auxílio de um sistema de informação, possibilitando assim o fácil acesso aos critérios de qualidade que devem ser verificados na conclusão dos pacotes de trabalho e o armazenamento dos dados coletados no canteiro de obras. Para isso foi proposta uma modelagem de dados para descrever os dados a serem armazenados no sistema de informação e suas relações. Também foram identificadas as entidades do banco de dados que precisavam constar na interface de coleta de dados, sendo assim criado um módulo de campo. No entanto, o sistema de informações utilizado tanto para o banco de dados como para o módulo de campo era apenas um protótipo para avaliação.

A terceira etapa do estudo empírico consistiu na aplicação da segunda versão do modelo de controle integrado. A aplicação foi realizada durante um mês, com um total de 22 dias de coleta de dados. As coletas duravam de 25 a $120 \mathrm{~min}$, dependendo da quantidade de pacotes de trabalho que estavam sendo realizados. Nessa etapa do estudo o modelo foi aplicado inicialmente pela própria pesquisadora e depois por outros pesquisadores para que a aplicabilidade do modelo pudesse ser avaliada. Nessa etapa de aplicação do modelo foram coletados dados relacionados à ocorrência de perdas por making-do, execução de pacotes informais, conclusão de pacotes planejados e verificação da qualidade dos serviços. Os dados coletados a partir da aplicação do modelo de controle integrado foram analisados pela pesquisadora e apresentados para os gestores da obra, a fim de avaliar a utilidade do artefato.

Durante a aplicação o modelo continuou a passar por refinamentos sempre que alguma possibilidade de melhoria fosse identificada. Depois de algumas melhorias foi realizada uma reunião com os gestores da obra para apresentar o modelo proposto. Participaram da reunião, além dos pesquisadores envolvidos na aplicação do modelo, os gerentes de planejamento e de qualidade da empresa e o engenheiro da obra. O objetivo da reunião era que os gestores, além de conhecer o modelo de controle integrado, pudessem sugerir melhorias, a fim de facilitar uma futura aplicação pela equipe da obra. Após a reunião, o gestor de qualidade e o engenheiro da obra acompanharam uma aplicação do modelo no canteiro de obras. As entrevistas abertas foram uma importante fonte de evidência nessa etapa, pois permitiram considerar a percepção dos gestores da obra na avaliação do modelo.

A última etapa do estudo empírico foi realizada com o objetivo de avaliar a aplicabilidade do artefato por terceiros, com o mínimo de intervenção da pesquisadora. Assim, o modelo de controle integrado foi aplicado por outros pesquisadores e também por potenciais usuários da empresa. A aplicação do modelo pelos pesquisadores foi realizada durante 5 semanas, totalizando 17 dias de coleta de dados. As aplicações tiveram duração de 30 a $90 \mathrm{~min}$, dependendo da quantidade de pacotes de trabalho em execução. Para a aplicação do modelo pela empresa foi realizado um treinamento com a

114 Leão, C. F.; Isatto, E. L.; Formoso, C. T. 
participação de duas pessoas do setor de gestão da qualidade. Nessa ocasião foi realizada uma apresentação detalhada do modelo e foi entregue um manual com todos os passos para sua utilização. Após o treinamento cada um dos usuários realizou uma aplicação do modelo no canteiro de obras, para avaliar as facilidades e dificuldades encontradas.

Após a aplicação do modelo foram realizadas entrevistas semiestruturadas com os pesquisadores envolvidos e com os usuários da empresa a fim de que eles relatassem as dificuldades em relação ao uso do modelo e também sugerissem melhorias. Essa avaliação foi apresentada aos gestores da obra em uma reunião para que esses pudessem fazer uma avaliação geral do modelo proposto. Os dados coletados no canteiro de obras a partir da aplicação do modelo também foram analisados pela pesquisadora, contribuindo na avaliação do artefato.

Para a avaliação do modelo de controle integrado foram propostos dois constructos: utilidade e aplicabilidade. O constructo utilidade está relacionado com a contribuição do modelo proposto para a gestão da obra, sendo desdobrado em três critérios:

(a) contribuição do modelo proposto para a percepção da necessidade de realizar o controle integrado da produção e da qualidade;

(b) contribuição do modelo proposto para a percepção da necessidade de monitorar pacotes informais e perdas por making-do; $\mathrm{e}$

(c) contribuição dos resultados para a definição de ações corretivas e preventivas para diminuir a ocorrência de perdas por making-do, retrabalho e excesso de trabalho em progresso.

Já o constructo aplicabilidade está relacionado com o uso do modelo proposto, sendo desdobrado em quatro critérios:

(a) facilidade de uso: foi aplicada uma entrevista semiestruturada aos usuários do modelo. Além disso, foi considerada para a avaliação deste subconstructo a observação participante da pesquisadora, porque ela acompanhou algumas aplicações do modelo realizadas pelos outros usuários;

(b) eficiência: refere-se ao tempo despendido na aplicação do modelo de controle integrado. Foi monitorado o tempo despendido na coleta e no processamento dos dados;

(c) adequação ao processo existente: foi avaliada sob duas perspectivas. Primeiro, foi avaliada a possibilidade de implementação do modelo em outras obras. No entanto, essa avaliação foi limitada, pois os dois empreendimentos nos quais o modelo foi implementado eram muito semelhantes. Foi também avaliada a possibilidade de inserção do modelo aos procedimentos da empresa. Para isso, na apresentação dos resultados do estudo para os gestores da obra, estes foram indagados sobre essa questão; e

(d) possibilidade de continuação do uso do modelo: foi observada a ocorrência de uso do modelo sem a interferência da pesquisadora. Além disso, na reunião de apresentação dos resultados, os gestores foram questionados sobre seu interesse em utilizar o modelo proposto em outras obras da empresa.

Pode-se apontar como limitações do processo de avaliação do modelo proposto o fato de que ele não foi aplicado pela própria empresa de forma continuada, por limitações de tempo e pelo fato de que o sistema informatizado desenvolvido consistia apenas de um protótipo.

\section{Proposta para o modelo de controle integrado}

O modelo de controle integrado é formado por um modelo de dados (representado pelo diagrama de relacionamento de entidades - ERD - na Figura 1) e um modelo de processo (Figura 2). Ambos foram testados através da aplicação de um protótipo simples, utilizando tablets.

O sistema de controle integrado foi concebido para operar sem a necessidade de rede, através de uma base de dados (BD) residente em um desktop (um servidor de BD) e uma base de dados no dispositivo móvel (para a coleta de dados). Para testar o modelo de dados proposto foram inicialmente criados bancos de dados nas plataformas $M y S Q L$ (para o servidor BD desktop) e Sqlite (para o dispositivo móvel), ambos com a mesma estrutura do modelo de dados. A base de dados do dispositivo móvel deve ser atualizada no início da semana, a partir dos pacotes de trabalho programados para a semana na reunião de planejamento de curto prazo, sendo que, ao final da semana, os dados coletados no período são transferidos para a base de dados do servidor. Segue-se então um processo de análise crítica dos dados e sua consolidação, para se dar início a um novo ciclo de planejamento e controle semanal.

Para o teste do modelo de dados, optou-se pelo uso de planilhas eletrônicas e arquivos no formato MS Excel tanto para o armazenamento das tabelas como para a interface para coleta de dados, ambos em um tablet com sistema operacional Android e tela de 7". 
Figura 1 - Diagrama ERD representando o modelo de dados

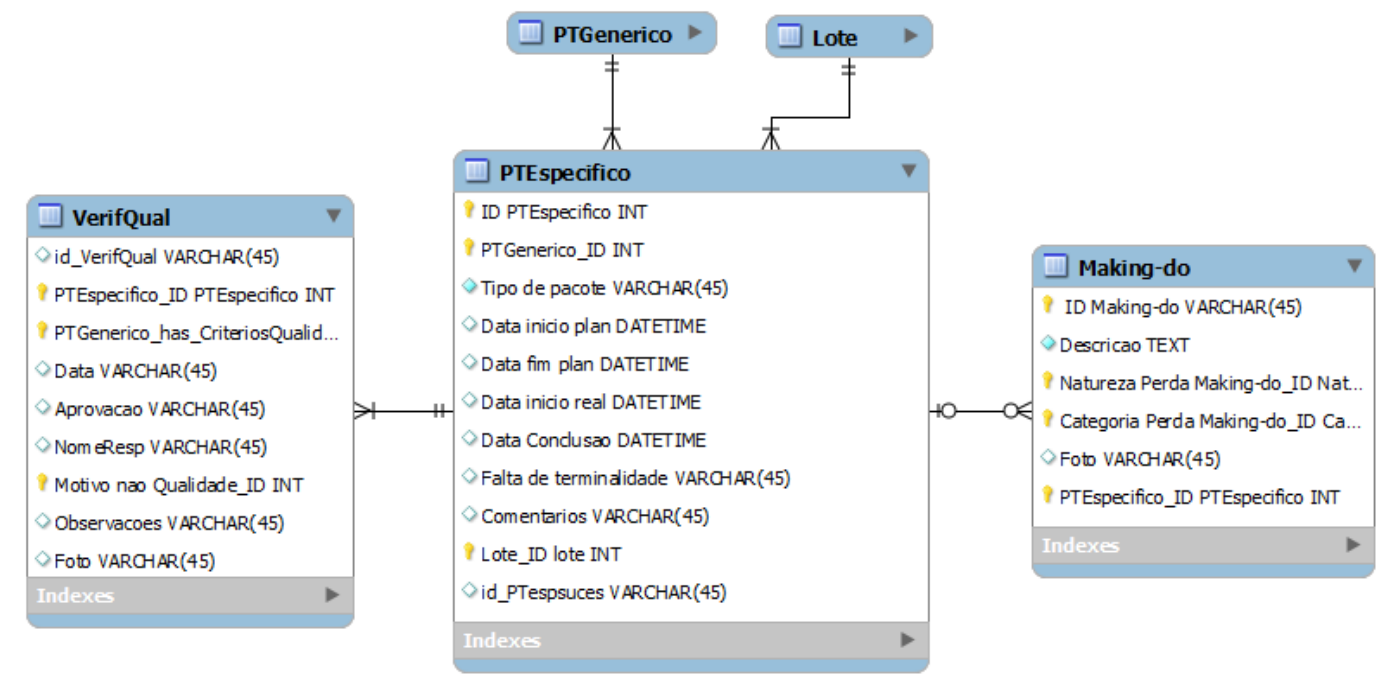

O protótipo construído para testar o modelo de controle integrado no canteiro de obras é formado por três planilhas eletrônicas, cada uma delas representando uma entidade do modelo de dados: uma planilha denominada "Pacotes" (Figura 3), composta dos pacotes de trabalho, representados no modelo pela entidade PTEspecífico; uma planilha denominada "Making-do" (Figura 4), destinada a registrar as ocorrências de perdas por making-do, que corresponde no modelo de dados à entidade de mesmo nome; e uma planilha denominada "Qualidade" (Figura 5), com as verificações da qualidade associadas com os pacotes e representada no modelo de dados pela entidade VerifQual.

Na planilha Pacotes (Figura 3) constam os pacotes de trabalho planejados para a semana e também são inseridos os pacotes informais identificados no canteiro de obras. A função dessa planilha é controlar a execução dos pacotes de trabalho, registrando as datas de início e de conclusão. O pacote de trabalho é a unidade de controle do modelo proposto. Denominado como PTEspecifico no modelo, ele deve corresponder a uma quantidade bem definida de serviço, a ser executado por um operário e equipe.

Seguindo a recomendação de Marchesan (2001), o modelo considera um pacote de trabalho como o resultado de uma ação aplicada em determinado elemento e um local específico. Neste caso, a ação representa um tipo de trabalho ou serviço a ser realizado (p. ex., realizar a concretagem), e o elemento representa uma parte bem definida da edificação (p. ex., paredes). Como as obras repetitivas se caracterizam pela existência de uma ou mais unidades de repetição (como, por exemplo, os vários modelos de casas), é natural que os pacotes de trabalho realizados em cada uma das casas também sigam um padrão de repetição, definido pelas possíveis combinações "ação + elemento" associadas ao modelo de casa. Tal combinação foi aqui considerada como um "pacote de trabalho genérico" (PTGenerico). Assim, o pacote de trabalho (PTEspecifico) pode ser visto como uma associação entre um pacote de trabalho genérico e um local específico, este último representado por uma ou mais instâncias da unidade-base (neste caso, as diferentes casas).

Na planilha Making-do (Figura 4), as perdas por making-do são classificadas conforme as categorias de improvisação propostas por Sommer (2010) e Fireman, Formoso e Isatto (2013), com pequenas alterações. No presente estudo, a categoria "ajuste de componentes" foi substituída pela categoria "materiais/componentes", devido ao fato de as perdas identificadas no estudo empírico estarem relacionadas com o uso de materiais ou componentes não adequados para a realização das tarefas. Também são identificadas as causas que deram origem às perdas por making-do, chamadas de natureza das perdas, conforme proposto por Sommer (2010). Além de incluir uma descrição do evento de making-do, é feito um registro fotográfico da improvisação e também é registrado em qual pacote de trabalho ocorreu o evento.

Já a planilha Qualidade (Figura 5) contém os critérios de qualidade que devem ser verificados em cada pacote de trabalho. Assim que o pacote é concluído, a data de conclusão aparece nessa planilha, demonstrando que o pacote de trabalho está liberado para a verificação da qualidade. Então, registra-se a data de verificação da qualidade, se o pacote foi aprovado (Sim/Não) e o nome do responsável pela verificação, podendo ainda ser feito um registro fotográfico dos problemas de qualidade. 
Figura 2 - Modelo do processo

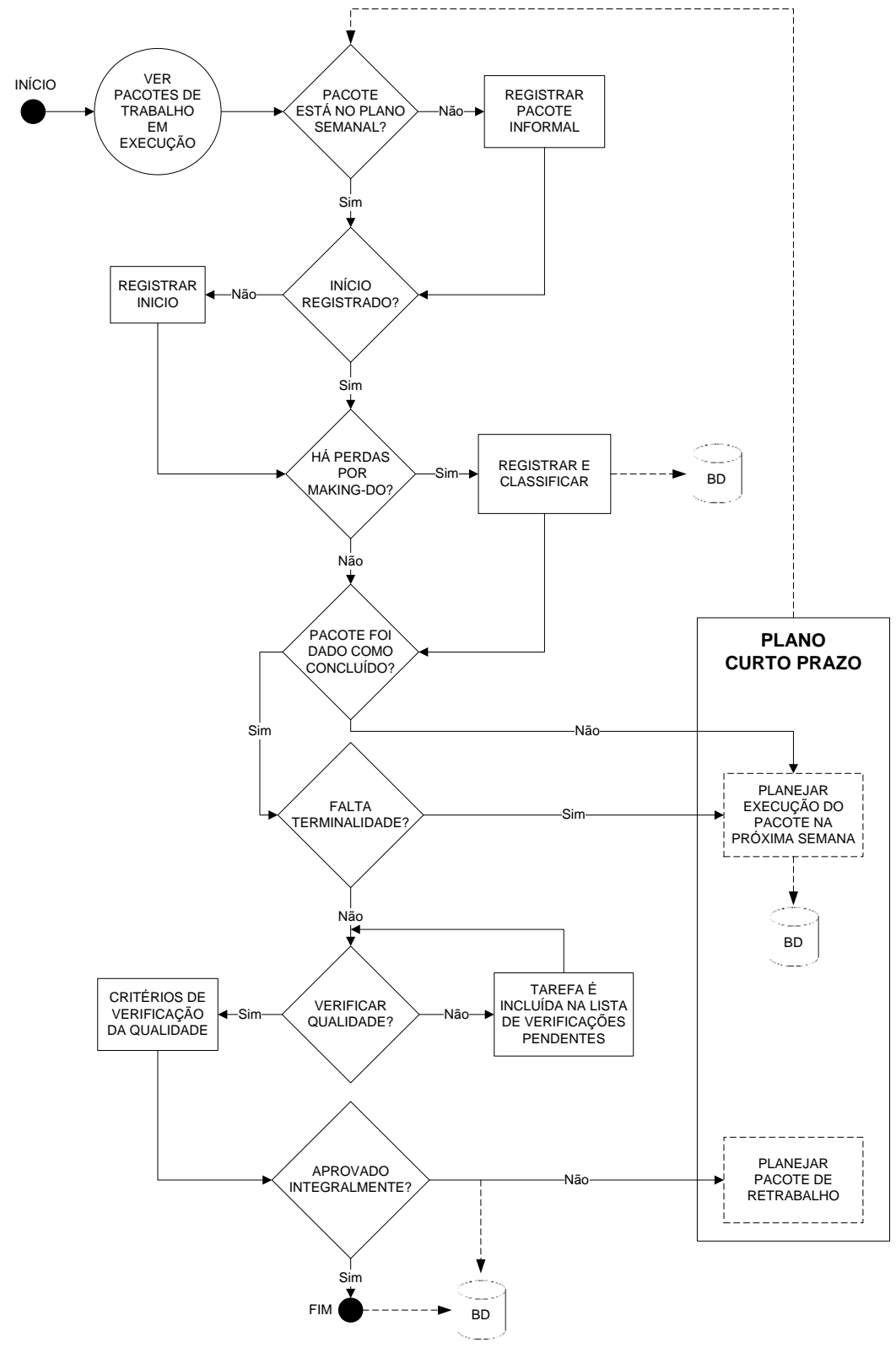

Figura 3 - Planilha Pacotes

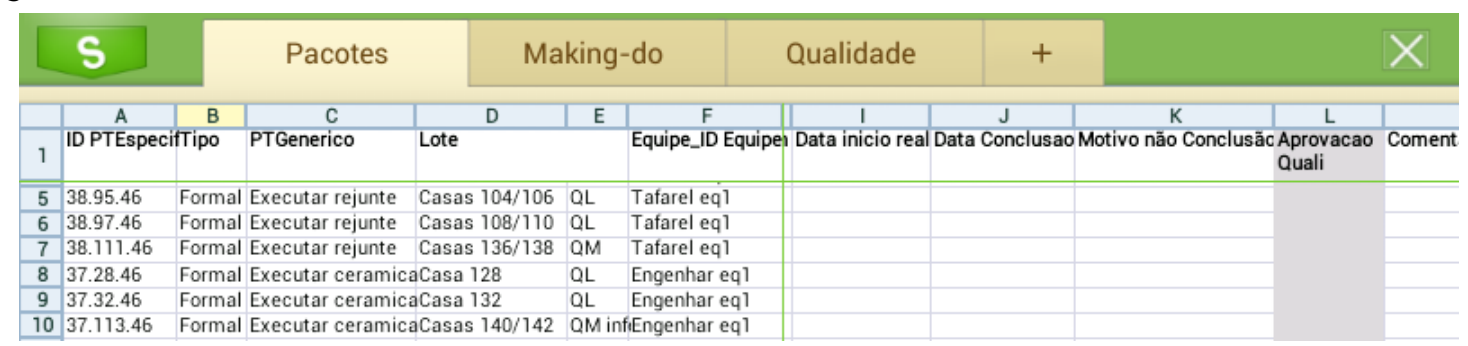


Figura 4 - Planilha Making-do

\begin{tabular}{|c|c|c|c|c|c|c|c|}
\hline & $\mathbf{S}$ & Pacotes & Making-do & Qualidade & + & & \\
\hline & A & B & C & D & $\mathrm{E}$ & $F$ & G \\
\hline 1 & ID & Making-do & Categoria & Natureza & Foto & ID_Ptesp & \\
\hline 2 & M01 & & & & & & \\
\hline 3 & M02 & & & & & & \\
\hline 4 & M03 & & & & & & \\
\hline
\end{tabular}

Figura 5 - Planilha Qualidade

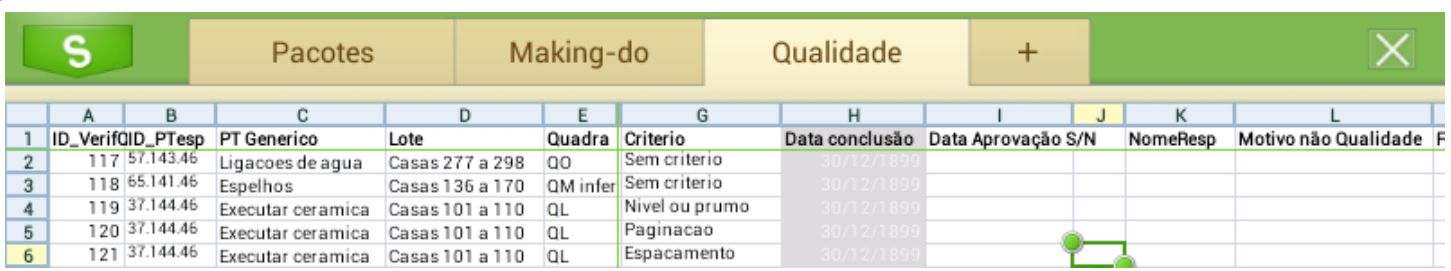

\section{Resultados}

Já em sua primeira aplicação o modelo de controle integrado permitiu identificar diversos pacotes informais sendo executados no canteiro de obras, as perdas por making-do de maior ocorrência e as dificuldades encontradas durante a verificação da qualidade dos pacotes de trabalho. Observou-se que o empreendimento possuía reduzida eficácia no processo de planejamento e controle, evidenciado pelo baixo PPC (media de 51\%) e pelo grande número de pacotes informais (34\%).

Durante a segunda aplicação do modelo de controle integrado, além das informações verificadas na primeira aplicação, foi também possível verificar a proporção dos pacotes executados com falta de terminalidade e avaliar a qualidade dos pacotes de trabalho concluídos, podendo ser calculados, além do PPC, os indicadores PPCQ e PPCR, propostos por Sukster (2005), e o indicador PPFT (porcentagem de pacotes com falta de terminalidade), calculado a partir da relação entre quantidade de pacotes de trabalho com falta de terminalidade e quantidade de pacotes planejados (Figura 6).

A existência de retrabalhos, por sua vez, contribuía para elevar o percentual de pacotes informais (PPI) executados na obra. Por exemplo, houve casos em que a execução do piso cerâmico foi dada como concluída no PPC, mas, ao avaliar a qualidade do serviço, foi identificada a presença de ocos entre o substrato e o revestimento cerâmico, causando descolamento ou quebra das peças, sendo necessário o retorno da equipe para refazer o serviço.
Quanto às perdas por making-do, foram identificadas as categorias de improvisação que tiveram maior ocorrência (Figura 7), assim como as causas (natureza) que deram origem a essas perdas (Figura 8) e os prováveis impactos (Figura 9). Por exemplo, constatou-se em diversas situações que as instalações elétricas ou hidráulicas não estavam totalmente concluídas com a qualidade necessária. Tais problemas de qualidade eram, muitas vezes, detectados pelas equipes subsequentes, tais como as de execução de forro de gesso ou pintura, quando estavam por iniciar seus serviços, estabelecendo uma condição de makingdo de natureza "serviços interdependentes". Como alternativa, as equipes recorriam à improvisação, realizando seu serviço mesmo sem condições de terminalidade, fazendo com que fosse necessário o retorno de uma equipe para posterior arremate, caracterizando assim uma improvisação na categoria "sequenciamento". Essa situação pode ser apontada como um exemplo típico de perdas por falta de terminalidade.

Além disso, a falta de uma rotina de reuniões de médio prazo contribuía para a existência das perdas por making-do. Como nessa etapa do estudo foram acompanhados os serviços referentes à fase final da obra, tais como revestimento cerâmico e pintura, foi possível observar a propagação de problemas em tarefas precedentes, tais como concretagem da parede e instalações elétricas e hidráulicas nos serviços de acabamentos. Essa propagação de defeitos foi observada pela porcentagem de pacotes de trabalho não concluídos com qualidade $(23 \%)$ devido à falta de qualidade na tarefa precedente. 
Figura 6 - Indicadores PPC, PPFT e PPCR

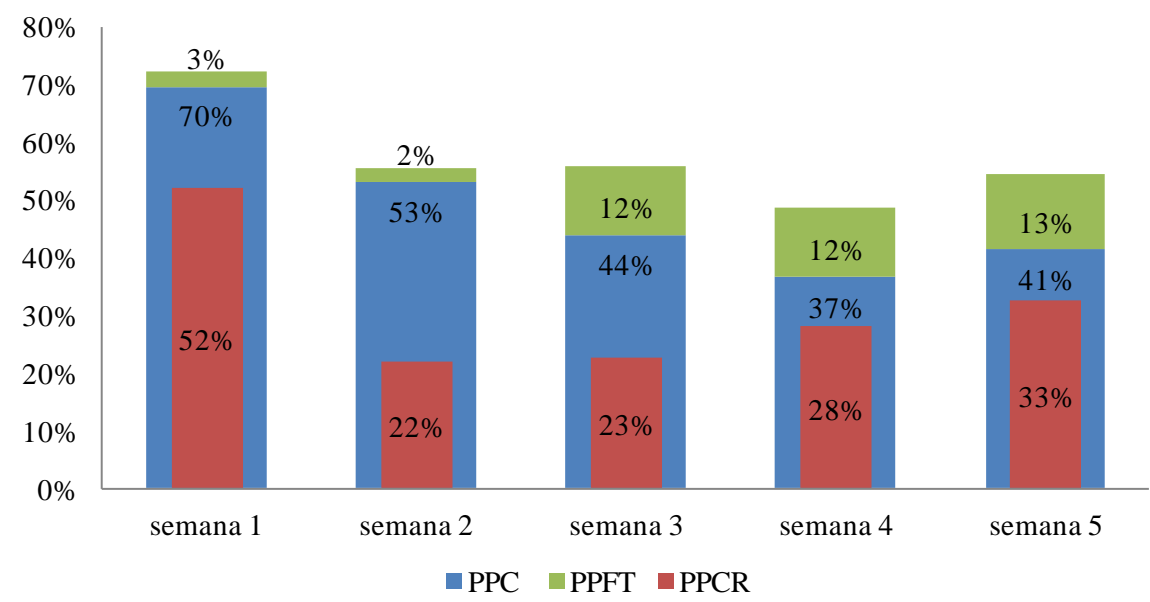

Figura 7 - Categorias de perdas por making-do na segunda aplicação do modelo

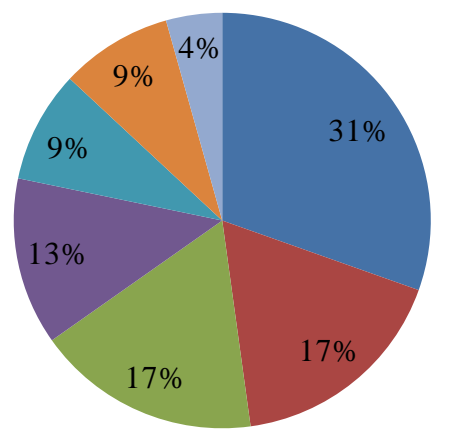

- sequenciamento

área de trabalho

- armazenamento

- equipamentos/ferramentas

- acesso/mobilidade

- instalações provisórias

materiais/componentes

Figura 8 - Natureza das perdas por making-do na segunda aplicação do modelo

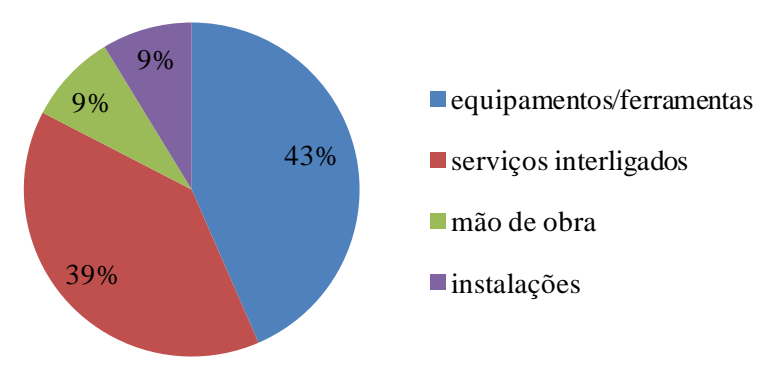

Figura 9 - Impacto das perdas por making-do na segunda aplicação do modelo

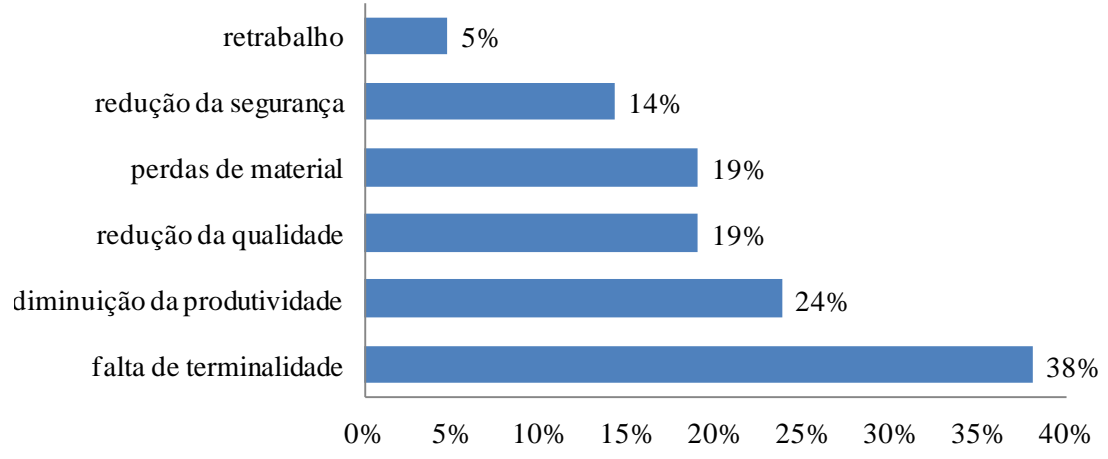

Proposta de modelo para controle integrado da produção e da qualidade com apoio da computação móvel 119 
Na última etapa do estudo empírico, o modelo foi aplicado por outros pesquisadores, que acompanharam os indicadores PPC e PPFT, além de identificar os pacotes informais executados no canteiro de obras. No entanto, os indicadores PPCQ e PPCR não puderam ser calculados, pois os pesquisadores encontraram dificuldades em avaliar a qualidade dos pacotes de trabalho executados, devido à falta de pessoal técnico com experiência nesse tipo de avaliação. Também foram identificadas as perdas por making-do durante a execução dos pacotes de trabalho. No entanto, os dados referentes a essas perdas não puderam ser analisados em profundidade devido à dificuldade de monitoramento dessas perdas por observadores pouco experientes, que não possuem muito conhecimento quanto ao procedimento de execução dos serviços.

\section{Utilidade do modelo}

No que se refere à contribuição do modelo para a percepção da necessidade de realizar o controle integrado da produção e da qualidade, essa necessidade foi percebida a partir da análise dos indicadores PPC, PPCQ e PPCR. Além de o PPC ser considerado baixo, o PPCR indicou que a porcentagem real de pacotes concluídos, ou seja, que não necessitam de um retorno da equipe para finalizar pendências ou problemas de qualidade, é ainda menor. Ao tomar conhecimento desses indicadores, os gestores mostraram-se surpresos, pois o procedimento de verificação da qualidade utilizado na empresa não mostrava os reais problemas de qualidade que ocorrem na execução dos serviços e que geram grande quantidade de retrabalho no canteiro de obras. Ainda, como os pacotes de trabalho não eram verificados logo após sua conclusão, os problemas propagavam-se para as tarefas seguintes, causando retrabalho não apenas na atividade de origem do problema, mas também nas tarefas posteriores. Isso não ocorreria caso a verificação da qualidade fosse realizada assim que o pacote de trabalho fosse concluído, permitindo realizar de forma imediata ações corretivas visando corrigir os problemas de qualidade identificados.

Quanto à contribuição do modelo para a percepção da necessidade de monitorar pacotes informais e perdas por making-do, pôde-se observar seu impacto na produção, gerando principalmente retrabalho e falta de terminalidade nas tarefas. As perdas por making-do da categoria sequenciamento estavam relacionadas com alguns dos problemas de qualidade identificados no canteiro de obras, o que indicava a necessidade de monitorar sua ocorrência para melhorar a qualidade dos pacotes de trabalho. Já no que diz respeito ao monitoramento dos pacotes informais, ficou evidente que tal monitoramento resulta em que esses pacotes sejam avaliados pelo controle da qualidade da mesma forma que os pacotes planejados, evitando assim que eventuais problemas de qualidade dos pacotes se propaguem para as demais tarefas.

No que se refere à contribuição dos resultados para a definição de ações corretivas e preventivas, os dados obtidos nas coletas de dados apontaram as deficiências na implementação do LPS na empresa. A falta de uma rotina de planejamento de médio prazo fez com que muitos pacotes de trabalho fossem planejados sem a remoção das restrições para sua execução. E, apesar da realização do planejamento semanal, esse não é utilizado pelos engenheiros da obra como um compromisso com o trabalho que deve ser realizado. Assim, os resultados do estudo empírico desencadearam maior interesse da empresa em melhorar a implementação do LPS nas obras. Com isso, foi iniciado um trabalho conjunto entre o setor de planejamento da empresa e a equipe de engenharia dos empreendimentos, no qual foram realizados treinamentos para a implementação das reuniões de médio prazo. Ainda, os gestores concordam que as informações coletadas no canteiro de obras com o modelo de controle integrado deveriam ser utilizadas nas reuniões de médio e curto prazo, auxiliando na gestão do empreendimento.

\section{Aplicabilidade do modelo}

Em relação à facilidade de uso, um fator fundamental que influencia na aplicação do modelo proposto é a experiência prévia do usuário em controle da produção e da qualidade. Por exemplo, é preciso ter conhecimento de quais tarefas estão incluídas nos pacotes de trabalho, para avaliar corretamente sua terminalidade. Também é necessário ter experiência na verificação da qualidade, pois alguns critérios são avaliados subjetivamente.

A eficiência do modelo proposto foi avaliada a partir da análise do tempo despendido na aplicação do modelo proposto no canteiro de obras durante a etapa 3 do estudo empírico. A avaliação foi baseada nessa etapa do estudo, pois se tratou de uma aplicação mais completa do modelo, na qual foram coletados dados relacionados tanto à conclusão e terminalidade dos pacotes quanto à verificação da qualidade, que é a atividade que despende maior quantidade de tempo. Foi monitorado o tempo despendido em 22 aplicações durante a etapa 3 do estudo, sendo o tempo médio de coleta de $65 \mathrm{~min}$, variando entre 25 e $120 \mathrm{~min}$, 
dependendo da quantidade de pacotes em execução e de pacotes concluídos, e da quantidade de verificações da qualidade realizadas. Por exemplo, em uma aplicação que durou $120 \mathrm{~min}$, dos 83 pacotes de trabalho planejados para a semana foram registrados nesse dia o início da execução de 16 pacotes e a conclusão de outros 20 pacotes, e ainda foi realizada a verificação da qualidade de 10 pacotes. Não foi verificada a qualidade de todos os pacotes concluídos porque alguns serviços requerem equipamentos específicos para avaliar alguns critérios de qualidade. Esses equipamentos ficavam armazenados no escritório da engenharia, localizado dentro do canteiro de obras, mas um pouco distante do local de coleta, devido à extensão do canteiro. Havia ainda a limitação de que o responsável na obra pela verificação da qualidade daquele serviço não estava presente no momento da coleta. Assim, alguns pacotes de trabalho concluídos tinham sua qualidade verificada nos dias subsequentes.

Apesar de não ter sido possível realizar uma comparação com o tempo despendido no procedimento atual de controle utilizado pela empresa, foram identificadas as vantagens do modelo proposto:

(a) compartilhamento do planejamento semanal entre os envolvidos no controle da obra, uma vez que no procedimento atual, muitas vezes, os pacotes de trabalho planejados são conhecidos no canteiro de obras apenas no final da semana, quando é verificada a conclusão deles, para calcular o indicador PPC;

(b) possibilidade de verificar a qualidade assim que o pacote de trabalho é concluído, pois os critérios de qualidade estão disponibilizados no tablet;

(c) compilação dos dados em um único local (tablet), o que reduz a quantidade de papéis que deve ser utilizada no canteiro de obras para realizar os controles, principalmente em relação ao controle da qualidade; $\mathrm{e}$

(d) com o uso de tablets para a coleta de dados, não há necessidade de reescrever as informações coletadas em cadernos e pranchetas, tornando as informações mais confiáveis e atualizadas.

Quanto à adequação ao processo existente, foi realizada uma avaliação em relação à possibilidade de implementação do modelo em outras obras e também à possibilidade de inserção do modelo nos procedimentos da empresa. Para avaliar a implementação em outras obras, o modelo foi aplicado em dois empreendimentos da empresa. No entanto, como se tratava de empreendimentos muito semelhantes, essa avaliação foi limitada.
Assim, como a empresa trabalha com outras tipologias, os gestores consideram importante avaliar a implementação do modelo em empreendimentos verticais e também em empreendimentos horizontais com padrão mais elevado, uma vez que os empreendimentos envolvidos neste estudo pertencem ao PMCMV. Essas outras tipologias diferem dos empreendimentos do estudo principalmente em relação ao sistema construtivo adotado, tipo de unidade repetitiva (casa, sobrado, apartamento ou pavimento), quantidade de pacotes de trabalho e tipos de pacotes informais executados. Essas características podem interferir no uso do conceito de pacote de trabalho genérico, que são aqueles pacotes que se repetem na unidade-base do empreendimento e também no controle da qualidade, em função do tamanho do lote de produção utilizado.

Quanto à possibilidade de inserção do modelo nos procedimentos da empresa, os gestores consideram viável, desde que ocorram algumas mudanças, tanto na empresa quanto no modelo. Em relação à empresa, é necessária a introdução de melhorias gerenciais mais amplas. Por exemplo, a empresa apresenta deficiências relacionadas à implementação do LPS, que precisam ser melhoradas para que o modelo de controle integrado possa ser eficaz e trazer benefícios. Já as mudanças necessárias no modelo estão relacionadas ao uso de TI. É preciso simplificar o sistema de informações, principalmente o módulo de campo, através do desenvolvimento de um aplicativo que facilite a coleta e a sincronização dos dados, podendo ser utilizado por qualquer usuário, mesmo por aqueles que não possuem conhecimento e domínio de ferramentas de TI.

Após a realização do trabalho, pode-se afirmar que o uso da TI é essencial para a implementação do modelo de controle proposto, devido à quantidade de dados que devem ser coletados e processados. $\mathrm{Na}$ etapa 3 do estudo empírico, por exemplo, foram inseridos no modelo 391 pacotes de trabalho específicos, uma média de 78 pacotes por semana, que deveriam ser controlados diariamente. No entanto, devido ao fato de o sistema de informações utilizado tratar-se apenas de um protótipo para avaliação, não foi possível alcançar os benefícios que a TI poderia fornecer, sendo necessário realizar melhorias na ferramenta utilizada.

\section{Conclusões}

A aplicação do modelo de controle integrado permitiu monitorar a conclusão de pacotes de trabalho, a execução de pacotes informais, perdas 
por making-do e falta de terminalidade dos pacotes, além de avaliar a qualidade dos pacotes de trabalho concluídos, sendo possível calcular diferentes indicadores referentes à eficácia do planejamento (PPC), grau de integração da gestão da qualidade com o PCP (PPCQ e PPCR) e grau de formalização do PCP (PPI). No entanto, não foi possível observar a redução das perdas por making-do, retrabalho e trabalho em progresso, devido às deficiências da empresa na implementação do LPS, que não permitiram a realização de ações corretivas e preventivas para a redução das perdas identificadas.

Os resultados obtidos a partir da aplicação do modelo de controle integrado apontam a importância em realizar os planejamentos de médio e curto prazo, a fim de identificar e remover as restrições dos pacotes de trabalho, evitando, assim, a ocorrência das perdas por making-do. As informações geradas pela aplicação do modelo de controle integrado devem ser utilizadas nas reuniões do LPS, de maneira que os pacotes com falta de terminalidade e problemas de qualidade possam ser planejados, evitando a execução de pacotes informais, e que a identificação das perdas por making-do contribua para melhorar o processo de remoção de restrições. Ainda em relação à identificação das perdas por making-do, foi observada a dificuldade de monitoramento dessas perdas por observadores pouco experientes, devido ao não conhecimento do procedimento padrão de execução dos serviços e ao caráter subjetivo dessa análise.

Como limitações da pesquisa realizada, pode-se destacar o fato de que houve dificuldades na coleta de dados referentes à avaliação da qualidade dos pacotes concluídos (indicadores PPCQ e PPCR) e à categorização de perdas por making-do, o que restringiu algumas das análises quantitativas realizadas. Além disso, o modelo não pôde ser efetivamente testado no contexto real da empresa, sendo o mesmo aplicado de forma continuada somente pela equipe de pesquisa.

A partir das conclusões apresentadas, sugere-se para trabalhos futuros:

(a) aplicar o modelo de controle integrado proposto em outros empreendimentos, com tipologias diferentes, como, por exemplo, empreendimentos verticais, para avaliar sua aplicabilidade em outros contextos;

(b) utilizar as tecnologias BIM e Realidade Aumentada para visualizar os procedimentos de execução dos serviços, auxiliando na identificação das perdas por making-do; $\mathrm{e}$ (c) desenvolver ferramentas de TI, como aplicativos para tablet, que auxiliem na coleta de dados no canteiro de obras, para o controle integrado da produção e da qualidade.

\section{Referências}

ALVES, T. Diretrizes Para a Gestão dos Fluxos Físicos em Canteiros de Obras: proposta baseada em estudos de caso. Porto Alegre, 2000.

Dissertação (Mestrado em Engenharia Civil) Programa de Pós-Graduação em Engenharia Civil, Universidade Federal do Rio Grande do Sul, Porto Alegre, 2000.

BALLARD, G. The Last Planner System of Production Control. Birmingham, 2000. Thesis (Ph.D) - School of Civil Engineering, Faculty of Engineering, University of Birmingham, Birmingham, 2000.

BALLARD, G.; HOWELL, G. Shielding Production: essential step in production control. Journal of Construction Engineering and Management, v. 124, n. 1, p. 11-17, 1998.

BONESI, F.M. Avaliação do Impacto Provocado Pelos Processos Construtivos Racionalizados nas Perdas Por Making-Do. Porto Aelgre, 2014 Trabalho de Conclusão de Curso (Graduação em Engenharia Civil) - Departamento de Engenharia Civil, Universidade Federal do Rio Grande do Sul, Porto Alegre, 2014.

FIREMAN, M. C. T. Proposta de Método de Controle Integrado Produção e Qualidade, Com Ênfase na Medição de Perdas Por MakingDo e Retrabalho. Porto Alegre, 2012. Dissertação (Mestrado em Engenharia Civil) - Programa de Pós-Graduação em Engenharia Civil, Universidade Federal do Rio Grande do Sul, Porto Alegre, 2012.

FIREMAN, M. C. T.; FORMOSO, C. T.; ISATTO, E. L. Integrating Production and Quality Control: monitoring making-do and unfinished work. In: ANNUAL CONFERENCE OF THE INTERNATIONAL GROUP FOR LEAN CONSTRUCTION, 21., Fortaleza, 2013. Proceedings... Fortaleza: IGLC, 2013.

FORMOSO, C. T. et al. Material Waste in Building Industry: main causes and prevention. Journal of construction engineering and management, v. 128, n. 4, p. 316-325, 2002.

FORMOSO, C. et al. An Exploratory Study on the Measurement and Analysis of Making-Do in Construction Sites. In: ANNUAL CONFERENCE OF THE INTERNATIONAL GROUP FOR LEAN CONSTRUCTION, 19, Lima, 2011. Proceedings... Lima: IGLC, 2011. 
FORMOSO, C. T.; MOURA, C. B. Evaluation of the Impact of the Last Planner System on the Performance of Construction Projects. In: ANNUAL CONFERENCE OF THE INTERNATIONAL GROUP FOR LEAN CONSTRUCTION, 17, Taipei, 2009. Proceedings... Taipei: IGLC, 2009.

HOPP, W. J.; SPEARMAN, M. L. Factory Physics: foundation of manufacturing management. Chicago: Waveland, 1996.

HWANG, B. et al. Measuring the Impact of Rework on Construction Cost Performance. Journal of Construction Engineering and Management, v. 135, n. 3, p. 187-198, 2009.

KIM, C. et al. On-Site Construction Management Using Mobile Computing Technology.

Automation in Construction, v. 35, p. 415-423, Nov. 2013.

KIM, Y. S. et al. A PDA and Wireless WebIntegrated System For Quality Inspection and Defect Management of Apartment Housing Projects. Automation in Construction, v. 17, n. 2, p. 163-179, jan. 2008.

KIMOTO, K. et al. The Application of PDA as Mobile Computing System on Construction Management. Automation in Construction, v. 14, n. 4, p. 500-511, ago. 2005.

KOSKELA, L. Management of Production in Construction: a theoretical view. In: ANNUAL CONFERENCE OF THE INTERNATIONAL GROUP FOR LEAN CONSTRUCTION, 7, Berkeley, 1999. Proceedings... Berkeley: IGLC, 1999.

KOSKELA, L. Making-Do: the eighth category of waste. In: ANNUAL CONFERENCE OF THE INTERNATIONAL GROUP FOR LEAN CONSTRUCTION, 12, Copenhagen, 2004. Proceedings... Copenhagen: IGLC, 2004.

KOSKELA, L. J.; HOWELL, G. The Underlying Theory of Project Management Is Obsolete. In: PMI RESEARCH CONFERENCE, 2002.

Proceedings... 2002.

KOUSHKI, P. A; KARTAM, N. Impact of Construction Materials on Project Time and Cost in Kuwait. Engineering, Construction and Architectural Management, v. 11, n. 2, p. 126132, 2004.

LAUFER, A.; TUCKER, R. Is Construction Project Planning Really Doing Its Job? A critical examination of focus, role and process.

Construction Management and Economics, v. 5, n. 3, p. 243-266, dez. 1987.
LOVE, P.; LI, H. Quantifying the Causes and Costs of Rework in Construction. Construction Management and Economics, v. 18, n. 4, p. 479490, 2000.

LUKKA, K. The Constructive Research Approach. In: OJALA, L.; HILMOLA, O-P. (Eds.) Case Study Research in Logistics. Publications of the Turku School of Economics and Business Administration, Series B1, 2003.

MACHADO, R. L. A Sistematização de Antecipações Gerenciais no Planejamento da Produção de Sistemas da Construção Civil. Florianópolis, 2003. Tese (Doutorado em Engenharia Civil) - Programa de Pós-Graduação em Engenharia de Produção, Universidade Federal de Santa Catarina, Florianópolis, 2003.

MARCHESAN, P. R. C. Modelo Integrado de Gestão de Custos e Controle da Produção Para Obras Civis. Porto Alegre, 2001. Dissertação (Mestrado em Engenharia) - Programa de PósGraduação em Engenharia Civil, Universidade Federal do Rio Grande do Sul, Porto Alegre, 2001.

MAROSSZEKY, M. et al. Quality Management Tools For Lean Production: moving from enforcement to empowerment. In: ANNUAL CONFERENCE OF THE INTERNATIONAL GROUP FOR LEAN CONSTRUCTION, 10, Gramado, 2002. Proceedings... Gramado: IGLC, 2002.

MISFELDT, E.; BONKE, S. Quality Control in Lean Construction. In: ANNUAL CONFERENCE OF THE INTERNATIONAL GROUP FOR LEAN CONSTRUCTION, 12., Copenhagen, 2004. Proceedings... Copenhagen: IGLC, 2004.

NOURBAKHSH, M. et al. Mobile Application Prototype For On-Site Information Management in Construction Industry. Engineering,

Construction and Architectural Management, v. 19, n. 5, p. 474-494, 2012.

PICCHI, F. A.; AGOPYAN, V. Sistemas da Qualidade na Construção de Edifícios. São Paulo, 1993. Departamento de Engenharia de Construção Civil, BT/PCC/104, Boletim Técnico da Escola Politécnica da USP.

SANTOS, D. G. Modelo de Gestão de Processos na Construção Civil Para identi|Ficação de Atividades Facilitadoras. Florianópolis, 2004. Tese (Doutorado em Engenharia Civil) - Programa de Pós-Graduação em Engenharia de Produção, Universidade Federal de Santa Catarina, Florianópolis, 2004. 
SILVA, R. C. M. Avaliação das Perdas Por Improvisação no Processo de Produção em Canteiros de Obras: estudo de caso na cidade de Maceió/AL. Maceió, 2013. Trabalho de Conclusão de Curso (Graduação em Engenharia Civil) Curso de Engenharia Civil, Universidade Federal de Alagoas, Maceió, 2013.

SOARES, A. Diretrizes Para a Manutenção e o Aperfeiçoamento do Processo de Planejamento e Controle da Produção em Empresas Construtoras. Porto Alegre, 2003. Dissertação (Mestrado em Engenharia Civil) - Curso de Mestrado Profissionalizante da Escola de Engenharia, Universidade Federal do Rio Grande do Sul, Porto Alegre, 2003.

\section{SOMMER, L. Contribuições Para Um Método} de Identificação de Perdas Por Improvisação em Canteiros de Obras. Porto Alegre, 2010. Dissertação (Mestrado em Engenharia) - Programa de Pós-Graduação em Engenharia Civil, Universidade Federal do Rio Grande do Sul, Porto Alegre, 2010.
SUKSTER, R. A Integração Entre o Sistema de Gestão da Qualidade e o Planejamento e Controle da Produção em Empresas Construtoras. Porto Alegre, 2005. Dissertação (Mestrado em Engenharia Civil) - Programa de Pós-Graduação em Engenharia Civil, Universidade Federal do Rio Grande do Sul, Porto Alegre, 2005.

VELOSO, C. O. Perdas Por Improvisação em Obra e Sua Relação Com o Planejamento de Médio Prazo. Salvador, 2014. Trabalho de Conclusão de Curso (Graduação em Engenharia Civil) - Escola Politécnica, Universidade Federal da Bahia, Salvador, 2014.

YU, H. et al. Development of Lean Model for House Construction Using Value Stream Mapping. Journal of Construction Engineering and Management, v. 135, n. 8, p. 782-790, 2009.

Cibeli Ferrando Leão

Faculdade de Arquitetura e Urbanismo | Centro Universitário Ritter dos Reis | Rua Orfanotrófio, 555, Alto Teresópolis | Porto Alegre - RS - Brasil | CEP 90840-440 | Tel.: (51) 3230-3315 | E-mail: cibeli_leao@yahoo.com.br

\section{Eduardo Luis Isatto}

Departamento de Engenharia Civil, Escola de Engenharia | Universidade Federal do Rio Grande do Sul | Av. Osvaldo Aranha, 99, Prédio Castelinho, Centro | Porto Alegre - RS - Brasil | CEP 90035-190 | Tel.: (51) 3308-3959 | E-mail: isatto@ufrgs.br

\section{Carlos Torres Formoso}

Departamento de Engenharia Civil, Escola de Engenharia | Universidade Federal do Rio Grande do Sul | Tel.: (51) 3308-3518

E-mail: formoso@ufrgs.br

\section{Revista Ambiente Construído}

Associação Nacional de Tecnologia do Ambiente Construído

Av. Osvaldo Aranha, $99-3^{\circ}$ andar, Centro

Porto Alegre - RS - Brasil

CEP $90035-190$

Telefone: +55 (51) 3308-4084

Fax: +55 (51) 3308-4054

www.seer.ufrgs.br/ambienteconstruido

E-mail: ambienteconstruido@ufrgs.br 